\title{
Development Status of High-Thrust Density Electrostatic Engines
}

\author{
Michael J. Patterson ${ }^{*}$ and Thomas W. Haag ${ }^{\dagger}$ \\ NASA Glenn Research Center, Cleveland, Ohio, 44135 \\ John E. Foster \\ University of Michigan, Ann Arbor, MI, 48109 \\ Jason A. Young ${ }^{\S}$ and Mark W. Crofton ${ }^{* *}$ \\ The Aerospace Corporation, El Segundo, CA, 90245
}

\begin{abstract}
Ion thruster technology offers the highest performance and efficiency of any mature electric propulsion thruster. It has by far the highest demonstrated total impulse of any technology option, demonstrated at input power levels appropriate for primary propulsion. It has also been successfully implemented for primary propulsion in both geocentric and heliocentric environments, with excellent ground/in-space correlation of both its performance and life. Based on these attributes there is compelling reasoning to continue the development of this technology: it is a leading candidate for high power applications; and it provides risk reduction for as-yet unproven alternatives. As such it is important that the operational limitations of ion thruster technology be critically examined - and in particular for its application to primary propulsion - its capabilities relative to thrust density and thrust-to-power ratio be understood. This publication briefly addresses some of the considerations relative to achieving high thrust density and maximizing thrust-to-power ratio with ion thruster technology, and discusses the status of development work in this area being executed under a collaborative effort among NASA Glenn Research Center, The Aerospace Corporation, and the University of Michigan.
\end{abstract}

\section{Nomenclature}

$\begin{array}{ll}A_{b} & =\text { beam area, } \mathrm{m}^{2} \\ \text { AGI-Engine } & =\text { Annular-Geometry Ion-Engine } \\ \text { DMH-Engine } & =\text { Dual-Mode Hybrid-Engine } \\ d_{s} & =\text { screen electrode aperture diameter, } \mathrm{m} \\ \mathrm{EP} & =\text { electric propulsion } \\ F & =\text { thrust, } \mathrm{N} \\ F_{A} & =\text { thrust density, } \mathrm{N} / \mathrm{m}^{2} \\ g & =\text { acceleration due to gravity, } \mathrm{m} / \mathrm{s}^{2} \\ \text { GRC } & =\text { Glenn Research Center } \\ \mathrm{HET} & =\text { Hall-Effect Thruster } \\ I_{a} & =\text { accelerator electrode impingement current, A } \\ I_{b} & =\text { beam current, A } \\ I_{b p s} & =\text { beam power supply current, A } \\ I_{d} & =\text { discharge current, A } \\ I_{n k} & =\text { neutralizer keeper current, A }\end{array}$

\footnotetext{
* Sr. Technologist, Propulsion Division, 21000 Brookpark Road/MS 301-3, AIAA Sr. Member

${ }^{\dagger}$ Aerospace Engineer, Space Propulsion Systems Branch, 21000 Brookpark Road/MS 86-8, AIAA Member

\# Associate Professor, Department of Nuclear Engineering and Radiological Sciences, 1906 Cooley Building 2355 Bonisteel Boulevard, AIAA Member

${ }^{\S}$ Member of Technical Staff, Space Materials Laboratory, M2-341, P.O. Box 92957, Los Angeles, CA 90009

** Sr. Scientist, Space Materials Laboratory, M2-341, P.O. Box 92957, Los Angeles, CA 90009, AIAA Sr. Member
} 


$\begin{array}{ll}I_{s p} & =\text { specific impulse, seconds } \\ J_{b} & =\text { ion beam current density, } \mathrm{A} / \mathrm{m}^{2} \\ l_{e} & =\text { effective acceleration length, } \mathrm{m} \\ l_{g} & =\text { interelectrode gap, } \mathrm{m} \\ m & =\text { ion mass, kg } \\ \text { MAGI-Engine } & =\text { Multi-ring Annular-Geometry Ion-Engine } \\ \text { NEXT } & =\text { NASA's Evolutionary Xenon Thruster } \\ \text { NGEPT } & =\text { Next-Generation Electric Propulsion Thruster } \\ \text { NSTAR } & =\text { NASA Solar electric propulsion Technology Application Readiness } \\ \text { PG } & =\text { pyrolytic graphite } \\ P_{i n} & =\text { thruster input power, } \mathrm{kW} \text { (unless otherwise specified) } \\ q & =\text { ion charge, } \mathrm{C} \\ \mathrm{SOA} & =\text { state of the art } \\ \mathrm{TAC} & =\text { The Aerospace Corporation } \\ t_{s} & =\text { screen electrode thickness, } \mathrm{m} \\ \mathrm{UM} & =\text { University of Michigan } \\ V_{a} & =\text { accelerator electrode voltage, } \mathrm{V} \\ V_{b} & =\text { beam voltage, } \mathrm{V} \\ V_{b p s} & =\text { beam power supply voltage, } \mathrm{V} \\ V_{d} & =\text { discharge voltage, } \mathrm{V} \\ V_{n k} & =\text { neutralizer keeper voltage, } \mathrm{V} \\ V_{t} & =\text { total accelerating voltage, } \mathrm{V} \\ \alpha & =\text { thrust-loss correction factor due to doubly-charged ions } \\ \beta & =\text { thrust-loss correction factor due to beam divergence } \\ \varepsilon_{i} & =\text { discharge losses, W/A } \\ \varepsilon_{o} & =\text { permittivity of free space } \\ \gamma & =\text { total thrust-loss correction factor } \\ \eta_{u} & =\text { total propellant utilization efficiency } \\ & \end{array}$

\section{Introduction ${ }^{\dagger \dagger}$} HERE remain many technical barriers to the wider implementation of electric propulsion (EP). Some
examples:

- No single EP technology exists that is scalable in specific impulse $(1500-5000$ seconds) at power levels of interest to the community ( $\geq 5-10 \mathrm{~kW}$ ). There are evolutionary paths following the status quo to develop a suite of different EP thruster technologies to meet NASA and other future mission needs. But this approach requires a multitude of technology development efforts, qualification tests, and life-tests with each requiring years of maturation, and each presenting unique spacecraft integration challenges.

- There remain unresolved technical concerns associated with candidate technologies proposed for near-term primary propulsion applications; as for example, Hall-Effect Thruster (HET) ground/space performance correlations.

- There is no present credible EP technology approaches which are scalable to multi-100 kW power levels; an important capability to support future human exploration architectures.

- While the demand for a wide range of EP systems is significant, the resources for thruster development and qualification are overly constrained to address the competing point design solutions for the numerous applications.

- EP systems are costly to develop, qualify, and field. EP systems are generally designed and qualified as point designs targeted at a specific use for a specific spacecraft. When another user or application comes along substantial new effort must be performed. While the cost of a single thruster is typically in the low hundreds of thousands of dollars, qualification and integration of the EP system may cost tens of millions of dollars. Part of this issue lies in a lack of commonality in EP approaches among NASA, non-NASA government, and commercial users.

${ }^{\dagger}$ All concepts disclosed in the publication are either covered under U.S. Patent \#8,468,794 ("Electric Propulsion Apparatus", June 25, 2013), or are Patent-Pending filed under both U.S. and International Patent Applications. Assignee: United State Government.

2

American Institute of Aeronautics and Astronautics 
- EP systems customers are risk averse. This hinders the implementation of new technology. While an EP system may have flown on numerous spacecraft, a scaled version to higher power is considered a new technology and categorized as high risk.

High-thrust density electrostatic (ion) thrusters, of both conventional and non-conventional construct, may be able to ameliorate some or all of these barriers. The Next-Generation Electric Propulsion Thruster (NGEPT) approach $^{2}$ - a non-conventional design utilizing an annular geometry - has been proposed as one means by which these barriers might be addressed. It may be able to do so by:

- Enabling the scaling of ion thrusters to very high power (>100 kW) and high thrust densities at modest $I_{s p}$, while

- Employing a reconfigurable architecture (ref. Fig. 1) designed with intent to capture the broadest possible set of mission applications.

More generally, ion thruster technology has attributes which make it quite attractive in comparison to other EP thruster technologies. In particular, ion thruster technology:

- Offers the highest performance and efficiency of any mature EP thruster technology;

- Has by far the highest total impulse of any technology option, demonstrated at input power levels (up to $7 \mathrm{~kW}$ ) appropriate for primary propulsion

- Has been successfully implemented for primary propulsion in both geocentric and heliocentric environments, with excellent ground/in-space correlation of both its

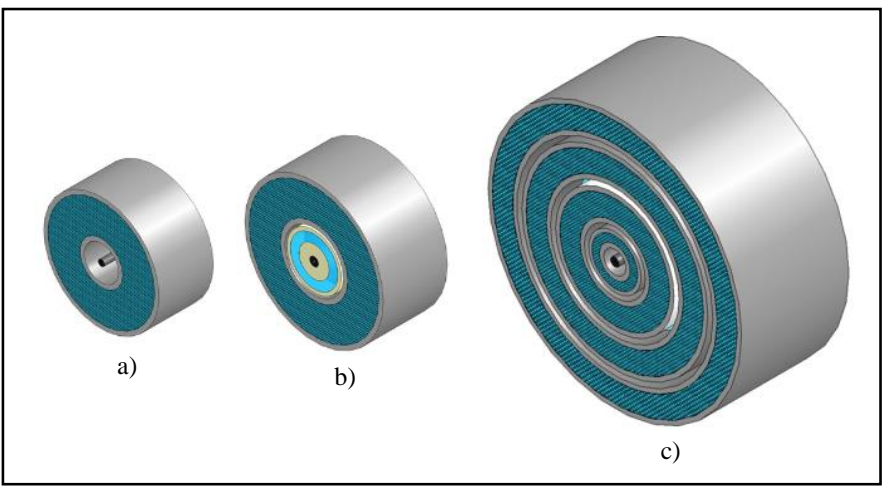

Figure 1. Potential NGEPT Configurations: a) an Annular Geometry Ion-Engine (AGI-Engine); b) a Dual-Mode Hybrid-Engine (DMH-Engine), comprised of an AGIEngine/HET Hybrid; and c) a Multi-ring Annular Geometry-Ion Engine (MAGI-Engine), from Ref. 2. performance and life.

What are generally characterized as weaknesses (or limitations) of ion thruster technology - its low thrust density/low thrust, and system complexity - might be more accurately characterized as attributes; attributes which have been driven by the mission application requirements imposed upon the technology to date. Illustrating this point:

- Ion thrusters are intentionally operated at low thrust densities for the purpose of achieving extremely long lifetimes needed to support the (typical) space science mission application. It is possible however to operate these devices at thrust densities and absolute thrust levels equal to that of other high power devices - e.g., more aligned with the requirements of earth-orbital needs. As an example, ion thrusters (nominal $30 \mathrm{~cm}$ beam dia.) were operated at $\sim 17 \mathrm{~kW} P_{i n}$, and $\sim 0.6 \mathrm{~N}$ thrust, corresponding to power and thrust densities of $\sim 330 \mathrm{~kW} / \mathrm{m}^{2}$ and $\sim 11.2 \mathrm{~N} / \mathrm{m}^{2}$ respectively, decades ago. ${ }^{3}$

- System complexity arises in the design implementation of the power processing and propellant management, which are in turn driven by the throttling requirements of the mission; specifically the dynamic ranges (input voltage, output voltage and power, and propellant flow rates). There is nothing inherent to ion thruster technology which makes the power conditioning or propellant flow control significantly more complicated than that which would be required for other EP thruster technologies, given the same imposed requirements relative to power and $I_{s p}$ operating condition, or ranges.

Based on these attributes there is compelling reasoning to continue the development this technology: it is a leading concept for high power applications; and it provides risk reduction for as-yet unproven alternatives. As such it is important that the operational limitations of ion thruster technology be critically examined - and in particular for its application to primary propulsion, its capabilities relative to thrust density and thrust-to-power ratio be understood.

This publication briefly addresses some of the considerations relative to achieving high thrust density and maximizing thrust-to-power ratio with ion thruster technology, and discusses the status of development work in this

\footnotetext{
\# “Ion Thruster Sets World Record,” July 28, 2013, http://www.nasa.gov/multimedia/imagegallery/image feature 2416.html
} 
area being executed under a collaborative effort among NASA Glenn Research Center (GRC), The Aerospace Corporation (TAC), and the University of Michigan (UM).

\section{Achie ving High Thrust Density}

This section discusses the limitations and reasonable expectations relative to achievable (near-term) thrust densities for electrostatic ion thrusters. This includes the constraints associated with both the ion optics and the plasma (discharge) source.

An electrostatic ion thruster thrust density, $F_{A}$, thrust per unit area in $\mathrm{N} / \mathrm{m}^{2}$, is given by:

$$
F_{A}=\gamma\left(2 \frac{m}{q}\right)^{0.5} I_{b}\left(V_{b}\right)^{0.5} / A_{b}
$$

Where $\gamma$ is the total thrust-loss correction factor, $m$ is the ion mass in $\mathrm{kg}, q$ is the ion charge in Coulombs, $I_{b}$ is the beam current in Amperes, $V_{b}$ is the beam voltage in volts, and $A_{b}$ is the active beam area in $\mathrm{m}^{2}$. The total thrust-loss correct factor $(\gamma)$ is a product of a thrust-loss correction factor associated with doubly-charge ions $(\alpha)$, and a thrustloss correction factor due to off-axis vectoring $(\beta)$.

For a given specific impulse, $I_{s p}$, the thrust density is then proportional to the product of the total thrust-loss correction factor and the ion beam current density, $J_{b}$ :

$$
F_{A} \propto \gamma \cdot J_{b}
$$

Where thrust density is maximized as $\gamma$ approaches unity and $J_{b}$ is maximized.

\section{A. Optics Limitations}

The ion beam current density is established primarily by the current extraction capability of the thruster ion optics. The ion optics current density is described by the Child-Langmuir equation, a form of which is given by the following:

$$
J_{b}=\left(\frac{4 \varepsilon_{O}}{9}\right)\left(\frac{2 q}{m}\right)^{1 / 2} \frac{V_{t}^{3 / 2}}{l_{e}^{2}}
$$

Where $V_{t}$ is the total accelerating voltage in volts, and $l_{e}$ is the effective acceleration length (a modified version of the interelectrode gap) in meters. The effective acceleration length, $l_{e}$, is given by:

$$
l_{e}=\left[\left(l_{g}+t_{s}\right)^{2}+\left(\frac{d_{s}}{2}\right)^{2}\right]^{1 / 2}
$$

Where $l_{g}$ is the interelectrode gap, $t_{s}$ is the screen electrode thickness, and $d_{s}$ is the diameter of the screen electrode apertures. For xenon propellant the expression for ion optics beam current density reduces to:

$$
J_{b}=4.771 \times 10^{-9} \frac{V_{t}^{3 / 2}}{\left(l_{g}+t_{s}\right)^{2}+\left(\frac{d_{s}}{2}\right)^{2}}
$$

Where the current density is maximized as the total voltage is increased to the highest practical value (while staying below the threshold which would result in interelectrode breakdown), and $l_{g}, t_{s}$, and $d_{s}$ electrode geometric parameters are reduced to practical limits.

1. Operating Near the Perveance Limit

Equation (5) defines the perveance-limited beam current density for a given ion optics configuration and total voltage. Historically ion thrusters are not operated at-or-near this perveance limit. Originally this had been the case because of thruster development concerns regarding internal erosion of cathode potential surfaces and the concomitant capability of the technology to achieve high total impulse. This issue has been well addressed by SOA NASA ion thrusters which incorporate design features which both reduce the cathode potential surface area exposed to the discharge plasma, and enable operation at discharge potentials at-or-below the threshold potential for ion 
sputtering. The combination of these featured have enabled repeated demonstration of long life (30,000-50,000 hours) with negligible internal component wear.

However SOA NASA ion thrusters (NSTAR and NEXT) are still operated far from the perveance limit. This now is the case because the simultaneous imposition of a mission-driven (high) specific impulse at a fixed (maximum) thruster input (constrained by the anticipated spacecraft bus power; for NSTAR about $2.3 \mathrm{~kW}$ into the thruster and NEXT about $6.9 \mathrm{~kW}$ into the thruster) drives the beam current to a value far-below that which would otherwise be accommodated by the electrostatic capability of the ion optics.

As an example if we take the nominal geometric characteristics of the NEXT thruster ion optics (conservatively assuming a 'hot' interelectrode gap equivalent to the 'cold' gap), at its maximum total voltage associated with the standard throttle table $(2010 \mathrm{~V})$, from Eq. (5) a maximum beam current density of about $215.8 \mathrm{~A} / \mathrm{m}^{2} \mathrm{could} \mathrm{be}$ supported. However the maximum beam current density of the NEXT standard throttle table is only about 43.2 $\mathrm{A} / \mathrm{m}^{2}$, or about $20 \%$ of that which should be supportable by the ion optics.

This 'discrepancy' between the NEXT ion optics capability and the manner in which the NEXT ion thruster is operated (in conformance with the planetary science mission throttle table) is illustrated more clearly by presenting the thrust density versus specific impulse. In Fig. 2 the thrust density for the NEXT ion thruster is plotted as a function of specific impulse for the conditions associated with the standard throttle table ('NEXT Throttle Table'). The thrust density is determined from Eq. (1) where the active beam area, $A_{b}$, is calculated from the product of the area established by the diameter of the (circular) ion optics and the screen electrode ion optics transparency (nominally, 0.80). The specific impulse, $I_{s p}$, in Fig. 1 is calculated from:

$$
I_{s p}=\eta_{u} \gamma\left(2 q V_{b} / m\right)^{0.5}(1 / g)
$$

Where $\eta_{u}$ is the total propellant utilization efficiency, and $g$ is the acceleration due to gravity. As indicated the thrust density ranges from about $0.32 \mathrm{~N} / \mathrm{m}^{2}$ at 1410 seconds $I_{s p}$, to a maximum of about $2.9 \mathrm{~N} / \mathrm{m}^{2}$ at 4200 seconds $I_{s p}$.

Also plotted in Fig. 2 is the maximum thrust density vs. specific impulse that would be anticipated for the NEXT thruster ion optics ('NEXT Optics Capability') as predicted from the beam current density from Eq. (5). In this instance the maximum total voltage, ion optics geometry, and thrust loss correction factors are per NEXT. As indicated the predicted maximum thrust density capability of the NEXT ion optics greatly exceeds the thrust densities as sociated with the standard throttle table at any given specific impulse; by about a factor of 3 at the lowest specific impulse, and a factor of 5 at the highest $I_{s p}$, peaking at about $14.4 \mathrm{~N} / \mathrm{m}^{2}$ at 4000 seconds $I_{s p}$. An additional data point, documented for a $30 \mathrm{~cm}$ divergent-field thruster operating at high thrust density (from Ref. 3), is also included in the figure.

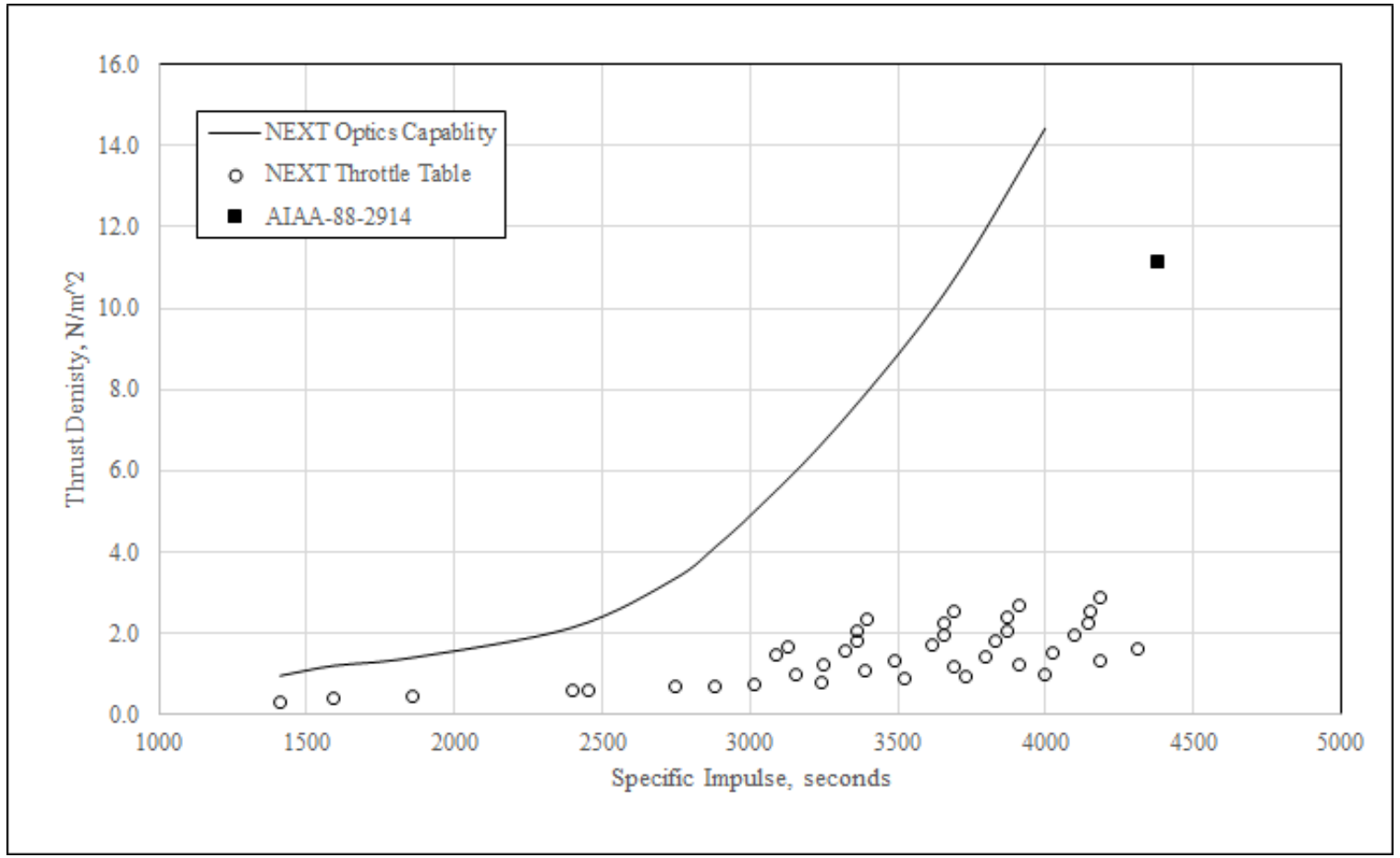

Figure 2. Thrust density versus specific impulse; NEXT ion optics. 


\section{2. 'Advanced-Design' Ion Optics}

Figure 2 indicates that substantial increases in thrust density, over that employed in typical throttling strategies, are achievable utilizing existing ion optics technology. While this appears to be the case, even further increases in maximum thrust density beyond those indicated in Fig. 2 might be enabled by improvements in the electrostatic design. This can be done by altering the NEXT optics electrostatics design.

Table 1 lists three potential design options: the standard NEXT optics design; a 'Version A' design which involves a reduction of the interelectrode gap, $l_{e}$, by $1 / 2$ and a reduction in the screen electrode thickness, $t_{s}$, to $2 / 3^{\text {rd }}$, $s$ that of the nominal value; and a 'Version B' which, in addition to an interelectrode gap $1 / 2$ that of nominal has screen electrode thickness of $1 / 3^{\text {rd }}$ nominal, and a very low thrust-loss correction factor associated with beam divergence (high $\beta=0.998$ ). In the case of a $2 \mathrm{X}$ reduction in interelectrode gap for both Version $\mathrm{A}$ and $\mathrm{B}$ the maximum supportable electric field strength is not exceeded at the NEXT total voltage of $2010 \mathrm{~V}$. In the case of the thinner screen electrodes for Version A ( $2 / 3^{\text {rd }}$ nominal) and Version B (1/3 $3^{\text {rd }}$ nominal), these values of $t_{s}$ are feasible for molybdenum and pyrolytic graphite (PG) respectively, having been demonstrated with these electrode materials. ${ }^{4}$ In the case of the high $\beta$ for Version $\mathrm{B}$, this has been demonstrated with flat (PG) electrodes on GRC's subscale Annular Engine. ${ }^{5}$ Also listed in Table 1 is the 'Perveance Enhancement' anticipated for Versions A (about 1.6) and B (about 1.8); that is, the increase in current density for these designs, over the (baseline) NEXT design, as predicted from Eq. (5).

Table 1. Optics Design Options

\begin{tabular}{|c|c|c|c|c|c|c|}
\hline \multirow{2}{*}{ Design } & \multicolumn{5}{|c|}{ Configuration } & \multirow{2}{*}{$\begin{array}{c}\text { Perveance } \\
\text { Enhancemen }\end{array}$} \\
\hline & $V_{t}$ maximu m, $\mathrm{V}$ & $l_{g}$ & $t_{s}$ & $\beta$ & $d_{s}$ & \\
\hline NEXT & \multirow{3}{*}{2010} & Nominal & Nominal & \multirow{2}{*}{ Nominal } & \multirow{3}{*}{ Nominal } & 1.000 \\
\hline Version $\mathrm{A}$ & & $1 / \mathrm{N}$ Nmingl & $2 / 3^{\text {rd }}$ Nominal & & & 1.595 \\
\hline Version B & & 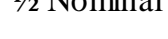 & $1 / 3^{\text {rd }}$ Nominal & 0.998 & & 1.784 \\
\hline
\end{tabular}

Figure 3 plots the logarithm of the thrust density, in $\mathrm{N} / \mathrm{m}^{2}$, versus specific impulse for the baseline (NEXT) ion optics design, along with design Versions A and B. For Version A the increase in thrust density relative to NEXT ion optics is simply equivalent to the increase in current density (the perveance enhancement); 1.60. For Version B the thrust density increase includes both the increase in current density and increase in $\beta$, yielding a factor of about 1.83 relative to NEXT.

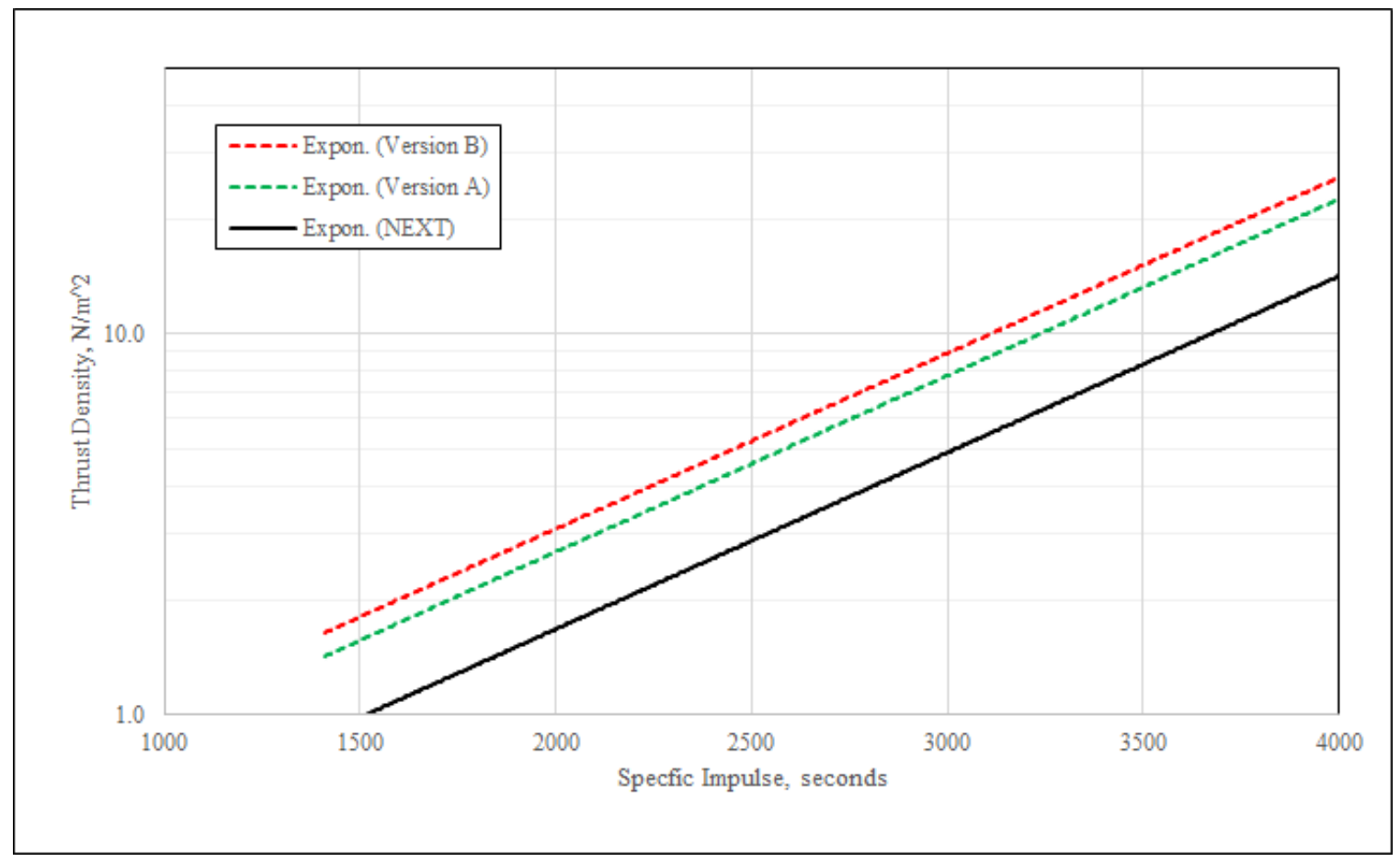

Figure 3. Thrust density versus specific impulse; NEXT and alternative ion optics designs. 6

American Institute of Aeronautics and Astronautics 
As indicated in Fig. 3, for Version A ion optics configuration - a straightforward modification (derivative) of conventional NEXT molybdenum optics - the peak thrust density varies from about $\sim 1.5 \mathrm{~N} / \mathrm{m}^{2}$ to $\sim 23 \mathrm{~N} / \mathrm{m}^{2}$ from 1410-4000 seconds $I_{s p}$. These thrust densities are quite high. A NEXT-diameter equivalent thruster, with Version A ion optics design, would produce peak absolute thrust levels of $\sim 0.15-2.3 \mathrm{~N}$ over $1410-4000$ seconds $I_{s p}$ range. For the highest perveance ion optics configuration, Version B, the peak thrust density varies from about $\sim 1.8 \mathrm{~N} / \mathrm{m}^{2}, \sim 3$ $\mathrm{N} / \mathrm{m}^{2}, \sim 5 \mathrm{~N} / \mathrm{m}^{2}$, and $\sim 9 \mathrm{~N} / \mathrm{m}^{2}$ at $1410,2000,2500$, and 3000 seconds $I_{s p}$ respectively, to $>26 \mathrm{~N} / \mathrm{m}^{2}$ at 4000 seconds $I_{s p}$. This design would likely require the implementation of PG in a flat geometry to achieve the indicated screen electrode thickness (with life) at high $\beta$.

Although these thrust densities are high - unless operation at conditions which exceed the Child-Langmuir equation can be demonstrated - to achieve absolute thrust levels in excess of 1 Newton at low ( $<3000 \mathrm{~s}) I_{s p}$ would require manufacturing of high-perveance optics with beam areas substantially greater than that of the $0.10 \mathrm{~m}^{2} \mathrm{NEXT}$ ion optics. Whereas, the manufacturing, mechanical, and thermal challenges associated with SOA sphericallydomed circular high-perveance ion optics designs impose a practical limit to the beam diameter of about $\approx 0.40 \mathrm{~m}$, equivalent to that of the NEXT ion thruster. Therefore to achieve higher thrust levels than about 0.15-2.3 Nover 1410-4000 seconds $I_{s p}$ range will require an alternative ion optics design approach to the spherically-domed circular geometry employed in SOA ion thrusters.

This situation presents some near-term challenges and opportunities to:

1. Investigate the indicated electrostatic design modifications to SOA (NEXT) ion optics to demonstrate enhanced thrust density;

2. Do so in a manner as not to compromise the life (total impulse) capability of the ion optics; and

3. Validate mechanical design approach(es) which enable manufacturing of high-perveance ion optics designs with active beam areas $>>0.10 \mathrm{~m}^{2}$, as may be required, to increase the absolute thrust levels at low $I_{s p}$.

Item (1), with the exception of improved $\beta$, can be addressed by altering NEXT optics electrostatics design. However this would not address items (2) and (3). To do so will require innovations in materials and mechanical design. Ultimately these achievable thrust densities may not be particularly useful unless long-life ion optics of $>0.10 \mathrm{~m}^{2}$ beam area can be built.

There has been little innovation in this technology as engendered by the SOA NSTAR and NEXT ion optics - in the electrostatic design, manufacturing processes, or materials - for nearly four decades. The referenced optics are almost indistinguishable in design with respect to the aforementioned characteristics as compared to ion optics developed in the late 70's for NASA's $30 \mathrm{~cm} \mathrm{Hg}$ ion thruster.

A notable exception in ion optics development is that proposed for implementation in the NGEPT design. ${ }^{4}$ The NGEPT design is intended to incorporate annular-geometry ion optics, manufactured from PG, potentially in a flat configuration (see Figure 4). This design approach would simultaneously address all 3 of the aforementioned challenges:

1. The machining of PG electrodes can be done with greater precision and with greater design flexibility than can be done with conventional electrode materials (e.g., molybdenum) by conventional manufacturing processes (e.g., chemical etching) thereby accommodating the implementation of advanced electrostatic designs;

2. The use of PG for the electrodes would yield an order-of-magnitude improvement in life capability relative to molybdenum which would more-than-offset the potential increase in thrust density and thereby ensure long life; and

3. The annular geometry would circumvent the limitations associated with conventional circular optics designs by enabling the manufacturing of very-large-area ion optics, while limiting the required electrode span and span-to-gap ratio.

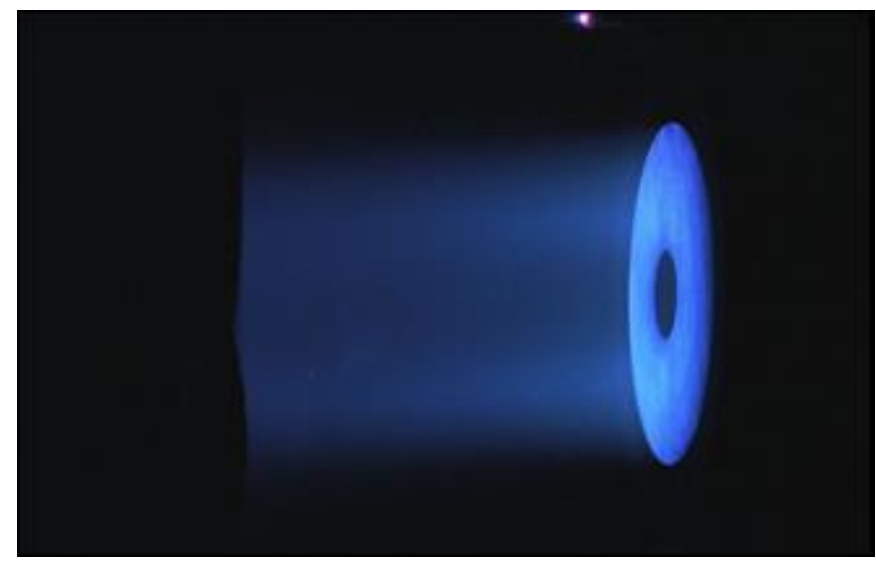

Figure 4. Sub-Scale AGI-Engine with flat PG electrodes operating under beam-extraction (from Ref. 4).

7

American Institute of Aeronautics and Astronautics 
Because of the novel method of fabricating large area ion optics, the NGEPT concept of an annular engine offers the prospect of operating at high thrust densities and high power. In this respect the annular engine may be the ultimate electric propulsion thruster - offering the best combination of high thrust and high specific impulse.

To date under the NGEPT development activity large-area ( 0.97 of the NEXT thruster beam area) flat PG annular ion optics of $46 \mathrm{~cm}$ O.D. and $5.1 \mathrm{~cm}$ I.D. have been fabricated and tested. ${ }^{4}$ These optics have demonstrated high perveance (comparable to NEXT optics), high $\beta$ ( 0.997), with a span-to-gap ratio less than $1 / 2$ that of the NEXT ion optics. This approach represents a promising forward avenue for addressing the identified challenges.

\section{Exceeding the Child-Langmuir Limit}

Conventionally, under the assumption of negligible initial energy, the maximum current density that can be extracted through the ion optics of an ion thruster is limited by the Child-Langmuir equation 3.

The space charge effects associated with this current flow essentially nullifies the electric field at the extraction surface thus setting a limit on current that can be injected into the interelectrode gap. Current flow higher than this value results in the formation of a potential barrier near the emission surface that would repel low energy ions flowing toward the interelectrode gap. For a given beam voltage, Eq. (2) therefore defines the upper thrust density limitation.

The form of the Child-Langmuir relation in Eq. (3) is actually a formulation associated with the special case in which injected ions leave the emission site at essentially zero initial velocity. Additionally, magnetic effects are not included. Inclusion of these processes generalizes the relation. It can be shown that in the most general formulation, the current density in fact can exceed that predicted by Eq. (3) by many factors ${ }^{6-9}$ Operating an ion thruster under such conditions could therefore in theory result in thrust density operation well beyond the upper limit predicted by the Child-Langmuir current density.

In general, there are at least three known conditions that can lead to interelectrode ion current densities exceeding the conventional Child-Langmuir limit. These include:

1. Introduction of counter-flowing negative charge in the interelectrode space;

2. Ion injection with a non-zero velocity at the emission site; and

3. Use of a magnetic mirror effect near the emission source.

The back flow of charged particles of opposite sign has the effect of neutralizing the interelectrode space charge, thereby extending the emission current limit, above which the potential gradient at surface is nullified. It is possible to generate the electrons (or negative ions) in the interelectrode space as well. For example, the interaction of an energetic ion beam with background gas under certain conditions can produce space charge neutralizing electrons. ${ }^{6}$ Alternatively electrons or negative ions could be allowed in a controlled manner to back-stream through the electrodes to achieve the same effect. Under either of these conditions, extractable ion current can be increased to arbitrarily high levels.

In the original formulation of the space-charge limit the ions are assumed to enter the interelectrode gap at zero velocity. This is of course a specialized case. Since the first derivations of the Child-Langmuir equation, the space charge limited current flow through a vacuum gap has been derived more generally to include arbitrary injection velocities as well as magnetic effects. When a non-zero injection velocity at the emission surface is taken into consideration, the generalized current density scales with the cube of the initial velocity. Qualitatively, the increased injection energy displaces the condition where the field is nullified to higher emission currents. In this regard, significant increases above the conventionally derived Child-Langmuir limit are possible. ${ }^{8}$

Finally, it has been shown that magnetic gradients can also extend maximum current levels beyond Child Langmuir. This involves conservation of the magnetic moment of the charged particle through the interelectrode gap. If the emission surface contains an axial magnetic field gradient in which the magnetic field decreases downstream, then conservation of the magnetic moment requires the charged particle increase its parallel velocity component as it drifts downstream, thereby circumventing the space charge generated barrier. ${ }^{9}$ This approach of course requires that the particles be magnetized in the region of the extraction zone.

While any of the aforementioned methods could be utilized in an ion thruster to increas e beam current density, counter-flowing charge or modification of initial injection energy are at present the most straightforward to implement that might be practically implemented in the longer term. Under these conditions then gridded ion thruster thrust densities could match or exceed those associated with HETs. HETs also have an upper limit on thrust density as well; when the magnetic field associated with the Hall current becomes comparable to the applied magnetic field, the device ceases to function as a Hall-Effect Thruster. 


\section{B. Source Limitations}

Other design constraints may limit ion thruster thrust densities to values below those predicted by operation at or near the Child-Langmuir equation shown in Fig. 3. One in particular appears to be the maximum supportable discharge (anode) current. ${ }^{4}$

The maximum thrust and thrust density demonstrated for the NEXT thruster is $0.466 \mathrm{~N}$ and $5.7 \mathrm{~N} / \mathrm{m}^{2}$ corresponding to an anode current of about 32 Amperes. At higher anode currents the discharge becomes highly resistive and unstable, indicating that there is insufficient anode surface area. In this instance then the maximum thrust and thrust density is limited by the source production - the ability to produce additional ion current - and not the ability of the optics to extract additional ion current. The effective anode surface area in a ring-cusp magnetic circuit discharge implemented in the NEXT thruster - the area which actively collects electron current from the plasma and sustains the discharge - is actually quite small relative to the total geometric surface area of anodepotential surfaces.

This behavior and performance is in stark contrast to that demonstrated with a much smaller ( $28.7 \mathrm{~cm}$ beam dia.) ion thruster which demonstrated a maximum thrust and thrust density of $0.577 \mathrm{~N}$ and $11.2 \mathrm{~N} / \mathrm{m}^{2}$ at an anode current of about 63 Amperes. ${ }^{3}$ This smaller thruster employed a nearly-identical electrostatic ion optics design as compared to that of the NEXT, but utilized a markedly-different magnetic circuit in the discharge chamber; a divergent field. This weakly-divergent field is used to contain the discharge plasma, but is much less effective in containing primary electrons than that used in SOA ion thrusters. However in this design the effective anode area available for electron collection should be equal to the total geometric surface area of anode-potential surfaces - which even for this small diameter thruster is much larger than the effective anode surface area of the NEXT ion thruster. So while the innovation of the ring-cusp magnet circuit design employed in SOA thrusters such NEXT has brought about significant improvements (high electrical efficiency, uniform plasma production, reduced electrode voltages and enhanced life) it may have done so at the expense of source production.

There are at least two ways to circumvent this source term limit, applied separately or in combination:

1. Incorporate the annular engine design approach of the NGEPT concept. This is because the annular discharge chamber increases the effective anode surface area for electron collection as compared to a conventional cylindrically-shaped ion thruster of equivalent beam area. This should enable operation at higher discharge currents and therefore high beam currents yielding an increase in thrust density, and if properly implemented, enabling ion optics operation at or near the Child-Langmuir limit.

2. Incorporate a divergent field magnetic design in the discharge chamber. This approach has the additional advantage that it typically employs permanent magnets which have much higher operating temperatures, and are much less susceptible to de-magnetization, as compared to the rare-earth magnets employed in SOA ringcusp designs. This is particularly important if the intent is to operate at higher thrust densities which will necessarily require operation at higher discharge power densities.

\section{Maximizing the Thrust-to-Power Ratio}

This section discusses the limitations and reasonable expectations relative to maximum thrust-to-power ratio for electrostatic ion thrusters. Producing high thrust is important. However doing in a power efficient manner, and as such in a competitive fashion relative to other EP thruster technology options, is also critically important.

\section{A. Analytical Solution}

The input power into an electrostatic ion thruster, $P_{i n}$, is given by:

$$
P_{i n}=\left(V_{b p s} I_{b p s}\right)+\left(V_{d} I_{d}\right)+\left(V_{n k} I_{n k}\right)+\left(\left|V_{a}\right| I_{a}\right)
$$

Where $V_{b p s}$ is the beam power supply voltage, $I_{b p s}$ is the beam power supply current, $V_{d}$ is the discharge voltage, $I_{d}$ is the discharge current, $V_{n k}$ is the neutralizer keeper voltage, $I_{n k}$ is the neutralizer keeper current, $V_{a}$ is the accelerator electrode voltage, and $I_{a}$ is the accelerator electrode impingement current.

For high input power levels, $P_{i n}$ can be simplified and approximated by:

$$
P_{i n} \cong I_{b}\left(V_{b}+\varepsilon_{i}\right)
$$

Where the discharge losses, $\varepsilon_{i}$, are the discharge power (W) required to produce an Ampere of beam ion current.

The thrust-to-power ratio, $F / P_{i n}$, then becomes: 


$$
F / P_{\text {in }}=\gamma\left(2 \frac{m}{q}\right)^{0.5}\left(V_{b}\right)^{0.5} /\left(V_{b}+\varepsilon_{i}\right)
$$

For xenon propellant this expression for thrust-to-power ratio reduces to:

$$
F / P_{\text {in }}=1.650 \times 10^{-3} \alpha \beta \frac{V_{b}^{1 / 2}}{\left(V_{b}+\varepsilon_{i}\right)}
$$

Where thrust-to-power ratio is maximized as the thrust-loss correction factors, $\alpha$ and $\beta$, approach unity, and the beam voltage, $V_{b}$, and the discharge losses, $\varepsilon_{i}$, are minimized.

\section{B. Performance Expectations}

For these calculations a fixed thrust-loss correction factor for doubly-charged ions, $\alpha$, is assumed equal to 0.9853 , corresponding to an (integrated) doubly-to-singly charged ion beam current ratio of 0.053 , which is typical of the NEXT ion thruster at full power. ${ }^{10}$ Two options (design solutions, reference Table 2) are considered: one being a SOA thruster configuration operating at high power; and a second being an advanced ion thruster design.

For the SOA thruster the assumption of operation at high power makes the approximate an alytical solution for $P_{i n}$, Eq. (8), valid. For the SOA thruster the thrust-loss correction factor due to beam divergence, $\beta$, is calculated in the manner described in Ref. 11, where spherically domed electrodes are assumed, with a dependency on the net-tototal voltage ('R') ratio. In this instance the discharges losses, $\varepsilon_{i}$, are assumed to be equal to $150 \mathrm{~W} / \mathrm{A}$, which is typical of the NEXT ion thruster at full power.

For the advanced ion thruster design flat ion optics electrodes are assumed, yielding a constant value for $\beta$ of 0.998; approximately equal to that which has been demonstrated with the flat annular PG ion optics on the subscale annular engine..$^{5}$ In this instance the discharge losses are assumed to be equal to $80 \mathrm{~W} / \mathrm{A}$. The value of $80 \mathrm{~W} / \mathrm{A}$ is a 'push goal.' The minimum cost for producing ions in the discharge chamber of an ion thruster (or any plasma source) can be estimated using Townsend's semi-empirical model for breakdown. ${ }^{12}$ For xenon gas the minimum energy cost for producing a xenon ion is approximately $37 \mathrm{eV}$. Some energy is also expended to produce and extract electrons, manifested as cathode heating and the extraction energy associated with the cathode fall voltage. Conservatively it can be assumed that the electron extraction energy is of order the discharge voltage; about $27 \mathrm{eV}$ per injected electron. Assuming a screen grid ion transparency of 0.80 and essentially no ion losses to anode surfaces, a value for $\varepsilon_{i}$ of $80 \mathrm{~W} / \mathrm{A}$ is then estimated.

Using the aforementioned assumptions the thrust-to-power ratio can be estimated versus specific impulse for the two cases, and these values are presented in Figure 5. Also plotted are previously-published data for a $30 \mathrm{~cm}$ thruster $^{13}$ and a NEXT ion thruster ${ }^{11}$ where this hardware was operated in a fashion to maximum the $F / P_{\text {in }}$ ratio. Additionally, data for a SOA HET, the BPT-4000, are included. ${ }^{14}$

In the case of the SOA thruster, identified in the figure as 'SOA Ion Thruster @ High Power', the $F / P_{\text {in }}$ ratio varies from a peak of about $60.2 \mathrm{mN} / \mathrm{kW}$ at about 1420 seconds specific impulse, to about $48.6 \mathrm{mN} / \mathrm{kW}$ at 2950 seconds $I_{s p}$. These projected values - which assume SOA technology but with a thruster configured specifically to achieve high $F / P_{\text {in }}$ operating at high power - show reasonable correspondence to data obtained on existing hardware operating at relatively modest power densities and without hardware modifications. These $F / P_{\text {in }}$ values down to about 1800 seconds $I_{s p}$ are comparable to those reported for the BPT-4000 HET.

In the case of the advanced thruster, identified in Fig. 5 as 'Advanced Ion Design', the F/Pin ratio varies from a peak of about $86.4 \mathrm{mN} / \mathrm{kW}$ at 1340 seconds $I_{s p}$, to about $53.5 \mathrm{mN} / \mathrm{kW}$ at 3000 seconds $I_{s p}$. These $F / P_{i n}$ values exceed those reported for SOA HETs ${ }^{14}$ over the entire specific impulse range, and do so for $\varepsilon_{i} \leq 120 \mathrm{~W} / \mathrm{A}$.

Table 2. Thruster Design Solutions

\begin{tabular}{|l|c|c|c|}
\hline \multirow{2}{*}{ Design } & \multicolumn{3}{|c|}{ Configuration } \\
\cline { 3 - 4 } & $\alpha$ & $\beta$ & $\varepsilon_{i}$, W/A \\
\hline SOA Ion Thruster @ High Power & \multirow{2}{*}{0.9853 (NEXT) } & Nominal (NEXT) & 150 \\
\cline { 1 - 2 } \cline { 3 - 4 } Advanced Ion Design & & 0.998 & 80 \\
\hline
\end{tabular}

10

American Institute of Aeronautics and Astronautics 


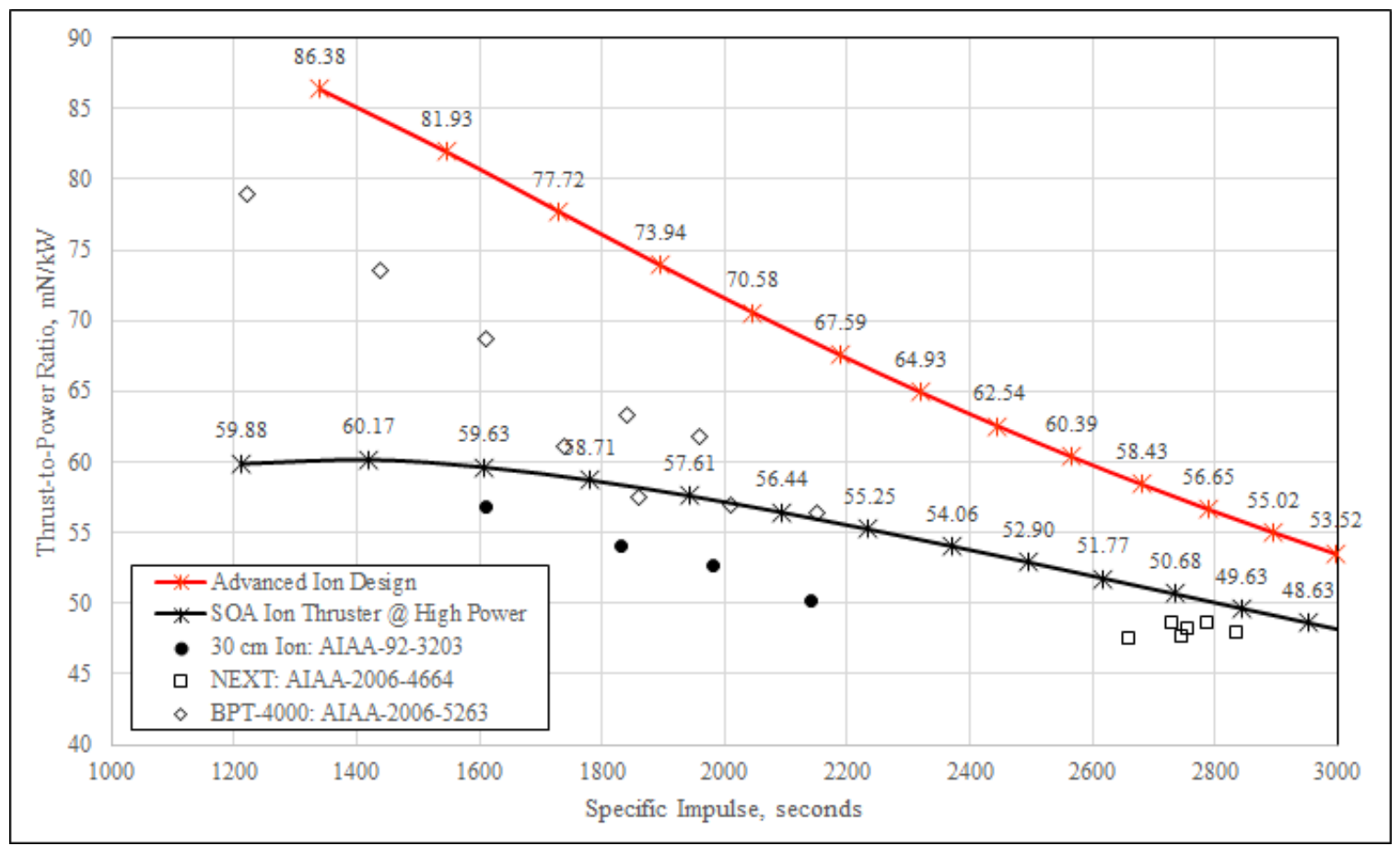

Figure 5. Thrust-to-Power ratio vs. specific impulse.

\section{Design Paths and Technology Development Status}

The aforementioned assessments have been considered in allocation of internal research and development resources among the participating organizations. Two paths appear to provide near-term opportunities for demonstration of high thrust density and high thrust-to-power ratio:

1. Continued development of the NGEPT concept, as manifested by the Annular-Geometry Ion-Engine; and

2. Demonstration of a (conventional) cylindrical-geometry ion thruster incorporating advanced-design ion optics and a magnetic circuit intended to inhibit source-limited operation.

These technology approaches, status, and other considerations are listed in Table 3. This section provides more detail with respect to these approaches and associated development status.

\section{A. Annular-Geometry Ion Engine}

The Annular-Geometry Ion Engine, or AGI-Engine, has many potential advantages over the SOA conventional ion thrusters. ${ }^{4,15}$ These include: the ability to scale to high power due to the novel ion optics design; the opportunity that the small span of the annular ion optics creates for the implementation of carbon electrodes with concomitant improvement in life time, performance (in perveance and $\beta$ ), and manufacturing processes; the increased anode surface area which inhibits source limiting, enabling operation at much higher thrust densities approaching the limit of the ion optics; and the potential to reconfigure the technology, and hybridize it with other EP thrusters, which could positively impact system performance and the cost to develop, qualify, and fly EP systems. Without the implementation of annular engine (or alternative) design approach to conventional spherically-domed cylindrical ion optics, there is little expectation that thrust levels exceeding about 0.15-2.3 N per thruster over the 1410-4000 second $I_{s p}$ range can be demonstrated. To this end resources have been committed to the development and demonstration of the annular engine.

11

American Institute of Aeronautics and Astronautics 
Table 3. Technology Approaches for High Thrust Density Ion Thrusters

\begin{tabular}{|c|c|c|c|c|c|}
\hline $\begin{array}{c}\text { Design/Technology } \\
\text { Approach }\end{array}$ & \multicolumn{2}{|r|}{ Status } & Near-Term Objectives & Concept Advantages & $\begin{array}{c}\text { Concept } \\
\text { Disadvantages }\end{array}$ \\
\hline \multicolumn{6}{|c|}{ NGEPT Approach - Annular-Geometry Ion-Engine } \\
\hline $\begin{array}{l}\text { 'GEN1' Annular- } \\
\text { Engine: Sub-Scale } \\
\text { Demonstrator } \\
\text { (40 cm dia.) }\end{array}$ & $\begin{array}{l}\text { Under } \\
\text { Test }\end{array}$ & $\begin{array}{l}\text { - GEN1A PG } \\
\text { Flat Optics } \\
\text { in Test } \\
\text { - GEN1B PG } \\
\text { Flat Optics } \\
\text { in } \\
\text { Fabrication }\end{array}$ & $\begin{array}{l}\text { Demonstrate: } \\
\text { - } F / P_{\text {in }}>\text { SOA HETs } \\
\bullet>86 \mathrm{~A} / \mathrm{m}^{2} J_{b} \\
\bullet>10 \mathrm{~N} / \mathrm{m}^{2} \mathrm{Max} .\end{array}$ & $\begin{array}{l}\text { - Provides Scalable } \\
\text { Pathway to Higher } \\
\text { Power } \\
\text { - Enables Application of } \\
\text { Advanced Optics } \\
\text { Electrostatics and } \\
\text { Materials to Enhance } \\
\text { Performance and Life } \\
\text { - Increased Anode } \\
\text { Surface Circumvents } \\
\text { Source Limited } \\
\text { Operation } \\
\text { - Enables } \\
\text { Reconfigurable } \\
\text { Architecture }\end{array}$ & \multirow[t]{2}{*}{$\begin{array}{l}\text { High Development } \\
\text { Cost for the Ion } \\
\text { Optics }\end{array}$} \\
\hline $\begin{array}{l}\text { 'GEN2' Annular- } \\
\text { Engine: Full-Scale } \\
\text { Demons tration } \\
\text { Engine } \\
\text { (65 cm dia.) }\end{array}$ & \multicolumn{2}{|c|}{ In Fabrication } & $\begin{array}{l}\text { Demonstrate: } \\
\text { - Scaling of Discharge and } \\
\text { Ion Optics } \\
\bullet F / P_{\text {in }}>\text { SOA HETs } \\
\bullet>86 \mathrm{~A} / \mathrm{m}^{2} J_{b} \\
\bullet \geq 20 \mathrm{~kW} P_{\text {in }} \\
\text { - Ion/HET Hybrid } \\
\text { Configuration } \\
\end{array}$ & $\begin{array}{l}\text { - Demonstrates Scalable } \\
\text { Pathway to Higher } \\
\text { Power }\end{array}$ & \\
\hline \multicolumn{6}{|c|}{ Conventional Approach - Cylindrical Engine } \\
\hline $\begin{array}{l}\text { Cylindrical } \\
\text { Engine: } \text { with } \\
\text { Divergent-Field } \\
\text { Magnetics } \\
\text { (40 cm dia.) }\end{array}$ & & Design & $\begin{array}{l}\text { Demonstrate: } \\
\text { - } F / P_{i n}>\text { SOA HETs } \\
\text { - }>10 \mathrm{~N} / \mathrm{m}^{2} \mathrm{Max} \text {. } \\
\text { - Up to } 8 \mathrm{~N} / \mathrm{m}^{2} \text { at } 3000 \mathrm{sec} \\
I_{s p} \\
\text { - Up to } 5 \mathrm{~N} / \mathrm{m}^{2} \text { at } 2500 \mathrm{sec} \\
I_{s p}\end{array}$ & $\begin{array}{l}\text { Provides Near-Term } \\
\text { High Power Thruster } \\
\text { Product with NEXT } \\
\text { Thruster Design } \\
\text { Heritage } \\
\text { - Increased Anode } \\
\text { Surface Circumvents } \\
\text { Source Limited } \\
\text { Operation }\end{array}$ & $\begin{array}{l}\text { - Does Not Provide } \\
\text { Design Pathway } \\
\text { to Higher Power } \\
\text { - Thrust Limited to } \\
\sim 0.2-2.3 \text { N over } \\
1410-4000 \text { sec } I_{s p}\end{array}$ \\
\hline
\end{tabular}

\section{1. 'GEN1' Annular-Engine}

To date a sub-scale demonstration engine, 'GEN1' depicted in Fig. 4, of $40 \mathrm{~cm}$ diameter incorporating flat ion optics manufactured from PG has been developed and is presently under test. ${ }^{4}$ This work was preceded by discharge chamber tests of the annular concept which were successful in demonstrating: stable annular discharge operation; uniform annular plasma densities using a singular discharge hollow cathode with an optimized magnetic circu it; and estimated discharge losses under conditions simulating beam extraction consistent with those values required to yield high overall engine efficiency. ${ }^{2}$ Subsequent to the discharge chamber tests the engine was retrofitted with conventional NEXT ion optics masked to simulate an annular geometry. During these tests with beam extraction, low discharge losses were demonstrated along with extremely-high beam current flatness values $(\sim 0.85-0.95){ }^{5}$

As reported in 2013 for the GEN1 engine: $40 \mathrm{~cm}$ diameter flat annular geometry electrodes of high-perveance design were successfully manufactured from PG; the PG electrodes were integrated with the annular discharge chamber, and the optics demonstrated relatively high perveance, with very-high collimation $(\beta \approx 0.997)$ and high beam flatness at multiple NEXT thruster throttle levels. ${ }^{4}$ The tests were impeded however by the lack of a mechanical mounting assembly for the ion optics electrodes, which resulted in occurrences of shorting, and potentially excessive electrostatic deflection of the electrodes. This issue is being addressed using two approaches.

The first approach is to improve the mechanical mounting scheme for the existing $40 \mathrm{~cm}$ flat PG electrodes (GEN1A) to improve the stiffness and eliminate implementation errors that resulted in high voltage shorting. The second approach is to manufacture new $40 \mathrm{~cm}$ flat PG electrodes (GEN1B) which incorporate design features to increase stiffness, vis-a-vis (tapered) electrode thickness variations. These second set of electrodes are presently

12

American Institute of Aeronautics and Astronautics 
under manufacturing at TAC. Both approaches will be integrated with the GEN1 engine discharge chamber for further test characterizations. Test objectives of which are to demonstrate operation at high beam currents, as well as document the optics perveance, the beam current density profiles, and the beam divergence. Results from these tests are anticipated to be published at a later date.

2. 'GEN2' Annular-Engine

Also as reported in 2013 to meet the objective of demonstrating scalability of the annular engine concept to high power - which includes scalability of the annular discharge chamber and ion optics, but also includes demonstrating higher supportable discharge currents and beam current densities than SOA ion thrusters - requires the fabrication of a full-scale annular engine. ${ }^{4}$ This 'GEN2' annular engine needs to be sufficiently large to have the potential to operate in the $\sim 10$ 's of $\mathrm{kW}$ power range, and establish whether or not the azimuthal and radial discharge and beam uniformity demonstrated with the GEN1 engine can be maintained using a single-cathode design. The larger size would also provide the opportunity to address manufacturing, as sembly, and test issues associated with larger-area PG electrodes, but purposely limited to $\leq 66 \mathrm{~cm}$ annulus outside diameter so that a single (monolithic) panel construction technique could be employed for the ion optics. The GEN2 would also be scaled to ensure that there would be sufficient anode surface to enable operation closer to the Child-Langmuir limit than is the case for conventional ion thrusters, while maintaining relatively-low discharge losses. With this scaling comes the opportunity to then assess whether or not higher discharge currents and higher beam current densities can be supported. The interior diameter of the annulus would be sufficient large to accommodate either a centrallymounted neutralizer cathode assembly or integration of a centrally-mounted HET to investigate a hybrid engine configuration.

The GEN2 annular engine is shown conceptually in Figure 6. The GEN2 design has beam dimensions of approximately $65 \mathrm{~cm}$ O.D. by $36 \mathrm{~cm}$ I.D., yielding a total (annular) beam area $>2 X$ that of NEXT ion thruster, with an anode area of $\sim 4 X$ that of NEXT. Both the discharge chamber and the ion optics are in manufacturing at GRC. The discharge chamber, shown in Figure 7, uses standard materials and construction methods employed for laboratory model thrusters. It is presently being configured with a ringcusp magnetic circuit. The ion optics electrodes, shown in Figure 8, are being manufactured from substrate nucleated PG panels. The electrodes will be flat but will incorporate design features to increase overall stiffness. The electrodes when completed will be secured to mounting rings fabricated from carbon fiber-reinforced carbon ('carboncarbon').

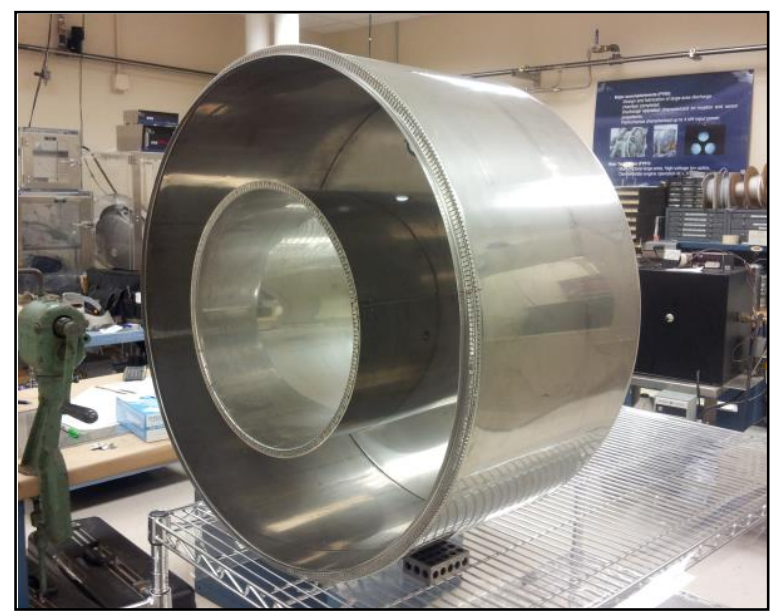

Figure 7. GEN2 Annular-Engine discharge chamber in fabrication.

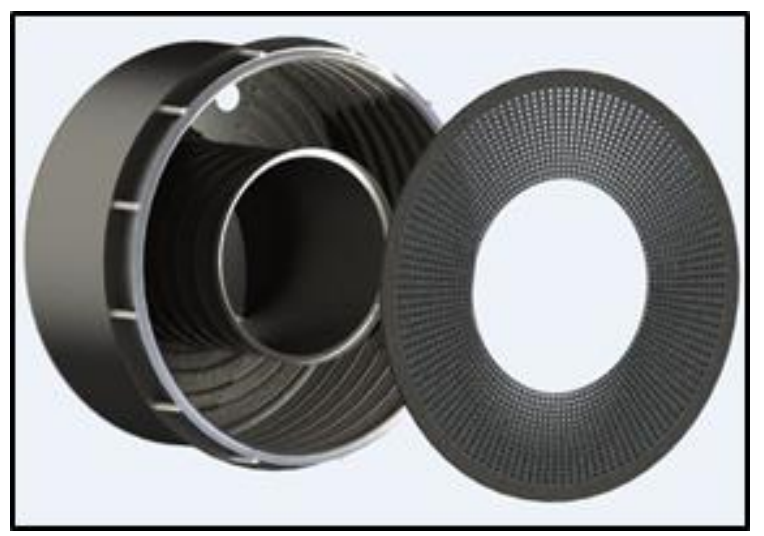

Figure 6. GEN2 Annular-Engine conceptual design.


Figure 8. GEN2 Annular-Engine Pyrolytic Graphite Ion Optics Undergoing Machining: Screen Electrode in Upper Image; Close-Up of Portion of Screen Eectrode in Lower Image.

13

American Institute of Aeronautics and Astronautics 
Upon completion the discharge chamber will be subjected to tests under conditions simulating beam extraction to: verify discharge stability; document discharge plasma azimuthal and radial uniformity; and estimate discharge losses. The discharge chamber will subsequently be mated to the ion optics assembly, and then subjected to a battery of tests to evaluate the functionality of the ion optics, and establish overall thruster performance and document the operational thrust and power envelopes, preliminary estimates of which are provided in Ref. 4.

With the GEN2 hardware as -built, the engine should be able to operate at:

- Thrust densities $\geq$ than that of 'Version A' ion optics identified in Figure 3; and

- Thrust-to-power ratios > than that of 'SOA' identified in Figure 5

At input power and thrust levels exceeding $20 \mathrm{~kW}$ and $1 \mathrm{~N}$ respectively.

\section{B. Cylindrical Engine}

Thrust and thrust densities of up to $0.58 \mathrm{~N}$ and $11.2 \mathrm{~N} / \mathrm{m}^{2}$ were previously demonstrated with a $30 \mathrm{~cm}$ divergentfield ion thruster on xenon propellant. ${ }^{3}$ Simply by replicating these input conditions a $40 \mathrm{~cm}$ NEXT-type ion thruster should be able to operate at thrust levels approaching 1 Newton. However as previously discussed this thruster is source limited, unable to support discharge currents yielding greater than about 7.0 Amperes beam current; about $0.47 \mathrm{~N}$. This is equivalent to about $86 \mathrm{~A} / \mathrm{m}^{2}$ beam current density, which is only about $40 \%$ of that predicted by the Child-Langmuir equation for NEXT ion optics.

One means of circumventing source limiting is to alter the magnetic circuit, doing so in a fashion as to increase the effective anode surface area while maintaining low discharge losses and without sacrificing source life. This should be feasible by implementing a divergent-field magnetic circuit configuration. This in combination with modifying the ion optics configuration to improve perveance - in this instance, starting from the NEXT ion optics design, and reducing the interelectrode gap and screen electrode thickness to mimic the configuration of the 'Version A' ion optics previously discusses - should enable the rapid development of a high thrust, high thrust density $40 \mathrm{~cm}$ ion thruster derived from the NEXT ion thruster. While this approach does not provide a pathway to very high power since it would not validate an ion optics design scalable beyond $\approx 0.10 \mathrm{~m}^{2}$, it may provide a nearterm technology product with thrust production capability up to $0.15-2.3 \mathrm{~N}$ over $1410-4000 \mathrm{~seconds} I_{s p}$, doing so at $F / P_{\text {in }}$ ratios comparable to SOA HETs. As such a $40 \mathrm{~cm}$ divergent-field thruster with an advanced ion optics configuration is in the design process.

\section{Summary}

This publication briefly reviewed some of the considerations relative to achieving high thrust density and maximizing thrust-to-power ratio with ion thruster technology, identifying limitations and reasonable expectations relative to achievable (near-term) thrust densities for electrostatic ion thrusters including the constraints associated with both the ion optics and the plasma source. The status of development work in this area collaboratively executed at NASA GRC, The Aerospace Corporation, and the University of Michigan was also reviewed.

SOA NASA ion thrusters are operated far from the ion optics perveance limit. This is the case because the simultaneous imposition of a mission-driven (high) specific impulse at a fixed thruster input drives the beamcurrent to a value far-below that which would otherwise be accommodated by the electrostatic capability of the ion optics. This results in a 'discrepancy' between the ion optics capability and the manner in which the ion thruster is operated, which is typically in conformance with the planetary science mission trajectory requirements.

For the NEXT ion thruster the predicted maximum thrust density capability of the ion optics greatly exceeds the thrust densities as sociated with the standard throttle table at any given specific impulse; by about a factor of 3 at the lowest specific impulse, and a factor of 5 at the highest $I_{s p}$ to about $14.4 \mathrm{~N} / \mathrm{m}^{2}$ at 4000 seconds. Even further increases in maximum thrust density might be enabled by straightforward alterations to the NEXT ion optics electrostatics design, raising peak thrust densities to about $\sim 1.5 \mathrm{~N} / \mathrm{m}^{2}$ to $\sim 23 \mathrm{~N} / \mathrm{m}^{2}$ over $1410-4000 \mathrm{~seconds}$ Isp range. More significant design alterations, such as flat electrodes manufactured from carbon, might yield thrust densities as high as $1.8 \mathrm{~N} / \mathrm{m}^{2}$ to $>26 \mathrm{~N} / \mathrm{m}^{2}$ over the same $I_{s p}$ range.

Although these thrust densities are high - unless operation at conditions which exceed the Child-Langmuir equation can be demonstrated - to achieve absolute thrust levels in excess of 1 Newton at low $(<3000 \mathrm{~s}) I_{s p}$ would require manufacturing of high-perveance optics with beam areas substantially greater than that of the $0.10 \mathrm{~m}^{2} \mathrm{NEXT}$ ion optics. Whereas, the manufacturing, mechanical, and thermal challenges associated with SOA sphericallydomed circular high-perveance ion optics designs impose a practical limit to the beam diameter of about $\approx 0.40 \mathrm{~m}$, equivalent to that of the NEXT ion thruster. Therefore, to achieve higher thrust levels than about $0.15-2.3 \mathrm{~N}$ over 1410-4000 seconds $I_{s p}$ range will require an alternative ion optics design approach to the spherically-domed circular geometry employed in SOA ion thrusters.

14

American Institute of Aeronautics and Astronautics 
This situation presents near-term challenges and opportunities to: (1) investigate electrostatic design modifications to SOA ion optics to demonstrate enhanced thrust density; (2) do so in a manner as not to compromise the life capability of the ion optics; and (3) validate mechanical design approaches which enable manufacturing of high-perveance ion optics designs with active beam areas $>>0.10 \mathrm{~m}^{2}$, to increase the absolute thrust levels at low $I_{s p}$. While modifications to the baseline NEXT ion optics can address the first challenge, implementation of the annular engine design concept incorporating annular-geometry ion optics manufactured from carbon would simultaneously address all 3 challenges by creating a viable engineering approach to manufacturing long-life large-area high perveance ion optics.

The annular engine concept would also circumvent the source limiting behavior of SOA ion thrusters - the condition where the maximum thrust and thrust density is limited by the source production (the ability to produce additional ion current) - and not the ability of the ion optics to extract additional ion current due to insufficient anode surface area. It does so because the annular discharge chamber increases the effective anode surface area for electron collection as compared to a conventional cylindrically-shaped ion thruster of equivalent beam area. This should enable operation at higher discharge currents and therefore high beam currents yielding an increase in thrust density, and if properly implemented, enabling ion optics operation at or near the Child-Langmuir limit.

Producing high thrust is important. However doing in a power efficient manner, and as such in a competitive fashion relative to other EP thruster technology options, is also critically important. For SOA ion thruster technology, thrust-to-power ratios from a peak of about $60.2 \mathrm{mN} / \mathrm{kW}$ at about 1420 seconds specific impulse, to about $48.6 \mathrm{mN} / \mathrm{kW}$ at 2950 seconds $I_{s p}$ should be feasible; projected values which show reasonable correspondence to data obtained on existing hardware operating at relatively modest power densities and without hardware modifications. These $F / P_{i n}$ values are comparable to those reported for SOA HETs down to about 1800 seconds $I_{s p}$. With improvements in discharge efficiency and ion optics $F / P_{\text {in }}$ ratios as high as about $86.4 \mathrm{mN} / \mathrm{kW}$ at 1340 seconds $I_{s p}$, to about $53.5 \mathrm{mN} / \mathrm{kW}$ at 3000 seconds $I_{s p}$ - values exceeding SOA HETs over the entire specific impulse range may be possible.

Two paths appear to provide near-term opportunities for demonstration of high thrust density and high thrust-topower ratio: (1) continued development of the annular engine concept; and (2) demonstration of a (conventional) cylindrical-geometry ion thruster incorporating advanced-design ion optics and a magnetic circuit intended to inhibit source-limited operation. The former provides a scalable pathway to higher power, while the latter may enable a nearer-term high power thruster product with NEXT design heritage.

Testing is underway to assess the performance of a sub-scale ( $40 \mathrm{~cm}$ diameter) 'GEN1' annular engine with pyrolytic graphite ion optics, intended to demonstrate beam current densities and thrust densities exceeding SOA. A 'GEN2' annular engine, with $65 \mathrm{~cm}$ beam outside diameter, is also in manufacturing. GEN2 will be used to demonstrate scalability (manufacturing, and operability) of the annular discharge chamber and ion optics, as well as demonstrating operation at beam current densities, thrust densities, and input power levels exceeding SOA.

\section{Acknowledgments}

The support of Mr. Jesus Lopez of Sierra Lobo, Inc. for design assistance and manufacturing of the pyrolytic graphite ion optics and Mr. Kevin McCormick of NASA GRC for thruster design assistance, fabrication, and assembly is gratefully acknowledged. This work was supported under the GRC Center Innovation Fund program sponsored by NASA's Office of Chief Technologist, and The Aerospace Corporation's Independent Research and Development Program.

\section{References}

${ }^{1}$ Crofton, M.W. and Pollard, J.E., “Thrust Augmentation by Charge Exchange," AIAA Paper No. 2013-4131, 49 ${ }^{\text {th }}$ Joint Propulsion Conference, July, 2013

${ }^{2}$ Patterson, M.J., Herman, D., Shastry, R., Van Noord, J., Foster, J.E., “Annular-Geometry Ion Engine: Concept, Development Status, and Preliminary Performance," AIAA Paper No. 2012-3798, $48^{\text {th }}$ Joint Propulsion Conference, July, 2012

${ }^{3}$ Patterson, M.J. and Rawlin, V.K., "Performance of 10-kW Class Xenon Ion Thrusters," AIAA Paper No. 88-2914, 24 ${ }^{\text {th }}$ Joint Propulsion Conference, July, 1988

${ }^{4}$ Patterson, M.J., Foster, J.E., Young, J.A., and Crofton, M.W., “Annular Engine Development Status,” AIAA Paper No. 2013-3892, 49th Joint Propulsion Conference, July, 2013

${ }^{5}$ Shastry, R., Patterson, M.J., Herman, D., Foster, J.E., "Current Density Measurements of an Annular-Geometry Ion Engine," AIAA Paper No. 2012-4186, 48 ${ }^{\text {th }}$ Joint Propulsion Conference, July, 2012

${ }^{6}$ Alexandrovich, I., "Space Charge Compensation of Technological Ion Beams," IEEE Trans. Plasma Science, Vol. 27, No. 4, August 1999, p. 1097 
${ }^{7}$ Feng, Yu-Cai and Wilbur, P.J. "Enhancement of Ion Beam Currents Through Space-Charge Compensation,” J. Appl. Phys., Vol. 54, No. 11, Nov. 1983, pp. 6113-6118

${ }^{8}$ Liu, S. and Dougal, R.A., "Initial Velocity Effect on Space Charge Limited Current," J. Appl. Phy s., Vol. 78, p. 5919, 1995

${ }^{9}$ Son, S. and Moon, S. J., "Overcoming the Child Langmuir Law Via the Magnetic Mirror Force," arXiV:1208.389 V1, 19 August, 2012

${ }^{10}$ Pollard, J.E, Diamant, K.D., Crofton, M.W., Patterson, M.J., and Soulas, G.C., "Spatially-Resolved Beam Current and Charge-State Distributions for the NEXT Ion Engine" AIAA Paper No. 2010-6779, 46 ${ }^{\text {th }}$ Joint Propulsion Conference, July, 2010

${ }^{11}$ Patterson, M.J., "NEXT Study of Thruster Extended Performance (NEXT STEP)," AIAA Paper No. 2006-4664, 42nd Joint Propulsion Conference, July, 2006

${ }^{12}$ Plasma Phy sics and Engineering, Alexander Fridman and Lawrence Kennedy, Ch. 4, Tay lor and Francis, NY, 2004

${ }^{13}$ Patterson, M.J., "Low-Isp Derated Ion Thruster Operation," AIAA Paper No. 92-3203, 28th Joint Propulsion Conference, July, 1992

${ }^{14}$ Welander, B., Carpenter, C., de Grys, K., Hofer, R.R., Randolph, D.H., and Manzella, D.H., "Life and Operating Range Extension of the BPT-4000 Qualification Model Hall Thruster," AIAA Paper No. 2006-5263, 42nd Joint Propulsion Conference, July, 2006

${ }^{15}$ Patterson, M.J., "Next-Generation Electric Propulsion Thrusters," AIAA Paper No. 2011-4812, 47th Joint Propulsion Conference, July, 2011 